\title{
Iron-dependent regulation of iron transporter expression in macrophages
}

\author{
E. Rose, R. Hoque and P. Sharp \\ Diabetes and Nutritional Sciences Division, King's College London, 150 Stamford Street, London, SE1 9 NH
}

Reticuloendothelial macrophages play a vital role in maintaining body iron homeostasis by recycling iron from haemoglobin following erythrophagocytosis, and scavenging free haem and haemoglobin from the circulation. Impaired processing of iron by macrophages, for example in inflammatory conditions ${ }^{(1)}$, can lead to the anaemia of chronic disease, characterised by macrophage iron loading. Currently our understanding of the mechanisms involved in macrophage iron transport and their regulation is incomplete. In this study we have investigated the effects of iron loading on the expression of the putative macrophage iron transporters, Nramp1 (Natural-resistance associated macrophage protein 1), DMT1 (Divalent Metal Transporter 1), and, TRPML1 and TRPML2 (Transient Receptor Potential Mucolipin 1 and 2).

Human THP1 cells were treated in vitro with 100 nM PMA (Phorbol 12-Myristate 13-Acetate) for differentiation into macrophages. The macrophages were treated with $50 \mu \mathrm{M}$ hemin or left untreated (control) for 24 hours at $37^{\circ} \mathrm{C}$. Changes in mRNA expression of Nramp1, DMT1, TRPML1 and TRPML2 were measured by real-time quantitative polymerase chain reaction and were normalised to the expression of $18 \mathrm{~S}$ mRNA.

\begin{tabular}{|c|c|c|c|c|}
\hline \multirow[b]{2}{*}{ Gene } & \multicolumn{2}{|c|}{ Control (arbitrary units) } & \multicolumn{2}{|c|}{$+50 \mu \mathrm{M}$ Hemin (arbitrary units) } \\
\hline & Mean & $\mathrm{SE}$ & Mean & $\mathrm{SE}$ \\
\hline Nrampl & 1.00 & 0.19 & $0.54^{\mathrm{a}}$ & 0.09 \\
\hline DMT1 & 1.00 & 0.36 & 0.97 & 0.37 \\
\hline TRPML1 & 1.00 & 0.14 & $0.61^{\mathrm{a}}$ & 0.08 \\
\hline TRPML2 & 1.00 & 0.13 & $0.57^{\mathrm{a}}$ & 0.07 \\
\hline
\end{tabular}

mRNA expression of macrophage iron transporters following exposure to hemin $(50 \mu \mathrm{M})$. Data are presented as mean and $\mathrm{SE} ; n=7$ in each group. Mean values, normalised to expression of $18 \mathrm{~S}$, were significantly different for those from control cells: ${ }^{a} P<0.05$ Student's $t$-test.

TRPML1 and TRPML2 are cation channels, expressed in macrophages, and have been shown to transport iron ${ }^{(2)}$. Here we show that their expression in human THP1 macrophages is decreased by addition of hemin, which suggests a potential role in macrophage iron metabolism. We also observed a decrease in Nramp1 expression following exposure to hemin. This is in contrast to previous findings in murine macrophages ${ }^{(3)}$, which may indicate some species-specificity in the regulation of Nrampl by iron. Interestingly, there was no significant effect of hemin on DMT1 expression; however, our experiments detected total DMT1 mRNA and further isoform-specific studies are needed to establish fully the role of iron in the regulation of $\mathrm{DMT}^{(4)}$ in macrophages.

In summary, the results of this study are consistent with roles for DMT1, Nramp1, TRPML1 and TRPML2 in macrophage iron transport; however, further studies are required to establish the precise function of these proteins in macrophage iron metabolism.

1. Weiss G (2009) Biochim Biophys Act 1790, 682-693

2. Dong X-P, Cheng X, Mills E et al. (2008) Nature 455, 992-996.

3. Soe-Lin S, Sheftel A, Wasyluk B et al. (2008) Exp Hematol 36, 929-937.

4. Hubert N and Hentze M (2002) Proc Natl Acad Sci USA 99, 12345-12350. 